\title{
Presheath Environment in Weakly Ionized Single and Multispecies Plasmas
}

\author{
Noah Hershkowitz, Fellow, IEEE, Eunsuk Ko, Student Member, IEEE, Xu Wang, Student Member, IEEE, and \\ Ahmed M. A. Hala
}

\begin{abstract}
The presheath located near boundaries in weakly ionized plasmas is a rich environment in which charge exchange, and ion-ion streaming instabilities combine to establish the electric fields that accelerate ions to close to the Bohm velocity at the sheath/presheath boundary. Charge exchange sets the presheath scale length in weakly collisional plasma, in which ionization can be neglected. The transition of mobility limited ion flow near the bulk plasma to free fall motion close to the plate for single species plasmas is explored. Measurements in argon-helium multidipole plasmas of plasma potential with emissive probes and ion energy distribution functions with laser induced fluorescence are presented. These data show that the argon ions are speeded up by the presheath electric fields, argon ions are heated, and ion-ion instability is present as ions approach the boundary.
\end{abstract}

Index Terms-Bohm velocity, charge exchange, free fall motion, ion-ion streaming instability, ion velocity, mobility limited flow, presheath, sheath, weakly ionized plasma.

\section{INTRODUCTION}

$\mathbf{S}$ HEATHS are nonneutral regions that form at the boundaries of plasmas. Langmuir [1] was the first to recognize that ions must have a finite velocity at the plasma/sheath boundary and suggested that ions derived their velocity from weak electric fields extending from the sheath edge some "considerable distance" into the plasma compared with the sheath thickness. This region is now known as the presheath. Bohm [2] showed that the minimum velocity of ions at the sheath edge of single species plasma with $T_{e} \gg T_{i}$ is the "Bohm velocity" $\sqrt{T_{e} / m_{i}}$, where $T_{e}$ is the electron temperature, $T_{i}$ is the ion temperature and $m_{i}$ is the ion mass. In some plasmas, the entire plasma provides ion acceleration and the presheath length is the plasma length. In many plasmas employed in industrial applications [3], and in basic plasma experiments, the ion-neutral collision length is small compared to the chamber dimensions and determines the presheath length. In such weakly ionized plasma, the presheath forms a well-defined region that can be distinguished from the bulk plasma.

Although presheath acceleration to a Bohm velocity has been a generally accepted phenomenon for some time, the first experimental measurements of the details of the electrostatic potential profile in a weakly ionized plasma were published only recently [4]. In the past, sheath phenomena were often emphasized while presheath phenomena were usually ignored. If considered at all, presheaths were only regarded as the region

Manuscript received September 23, 2004; revised December 11, 2004.

The authors are with the Center for Plasma-Aided Manufacturing, University of Wisconsin, Madison, WI 53706 USA (e-mail: hershkowitz@engr.wic.edu). Digital Object Identifier 10.1109/TPS.2005.844608 where ions were accelerated to the Bohm velocity. In this paper, we argue that much more is happening in the presheath. We show that the plasma presheath in weakly ionized plasma is a rich environment in which charge exchange, ion-ion streaming instabilities, and collisions combine to establish the electric fields that accelerate ions to close to the Bohm velocity at the sheath/presheath boundary and to determine the spatial profile of the ion temperature.

Weakly ionized gases are often approximated as collisionless plasmas because particle collision lengths are much greater than Debye lengths. Under this circumstance, plasma physicists tend to ignore the neutrals when considering sheath effects while recognizing they usually serve as the source of plasma ions and are often the dominant species. This approach has proved useful in studying a variety of phenomena such as linear and nonlinear waves. Collisions with neutrals can be treated as a small perturbation. What is sometimes not appreciated in fusion applications is that neutrals play an essential role in determining many zeroth order charged particle plasma parameters of partially ionized gases.

Plasma processing of materials depends on the interaction of ions and neutrals with surfaces. Ion energy distribution functions (IEDF) often play an important role in the processing. Electron-neutral collisions result in atomic/molecular excitation and ionization but usually do not provide significant ion heating in the bulk plasma. The IEDF at the substrate is determined by the ion acceleration in the plasma/substrate.

\section{THeORETICAL CONSIDERATIONS FOR Single ION PRESHEATH}

Sheath and presheath behavior are somewhat understood for single ion species plasma. Assuming ions are cold, the ion fluid equations can be written

$$
\begin{aligned}
& v \frac{d v}{d z}=\frac{q E}{\mathrm{~m}}-\nu_{c} v \\
& \frac{d}{d z}(n v)=0
\end{aligned}
$$

where $\nu_{c}=\langle\sigma v\rangle n_{0}$ is the ion-neutral collision frequency, $\sigma$ is ion-neutral collision cross section, and $\mathrm{n}_{0}$ is the neutral density.

A continuum of solutions exists for (1) and (2) depending on the relative sizes of the mean free path for collisions, the mean free path for ionization, and the chamber dimensions [5]-[8]. Here we consider an important subset of conditions: weakly ionized plasma in which ionization can be neglected. This regime is particularly important in plasma processing. Riemann has shown that close to the plasma boundary, when ionization can 
be neglected and when the ion-neutral collision cross section is either independent of $v$ or proportional to $1 / v$, the plasma potential in the presheath varies as

$$
\frac{e\left(\phi-\phi_{0}\right)}{T_{e}}=\sqrt{\frac{x-x_{0}}{\lambda_{c}}}
$$

where $x_{0}$ and $\phi_{0}$ are the position and potential of the sheath/ presheath boundary and $\lambda_{c}$ is the ion-neutral collision length [9]. Neglecting the convective derivative in (1) gives mobilitylimited flow with

$$
v=\mu E
$$

in the presheath, where $\mu$ is the ion mobility. However, the presheath potential described by (3) has an E field $\rightarrow \infty$ at the plasma/sheath boundary. So how fast do ions go near the plasma boundary where $E \rightarrow \infty$ ? It can be shown that there is a transition of mobility limited ion flow near the bulk plasma to free fall motion at the Bohm velocity close to the boundary for a single species plasma.

The plasma in the presheath can be assumed to be quasi-neutral with ion density $n_{i}$ approximately equal to electron density $n_{e}$. Neglecting the electron mass in the electron fluid equations gives the Boltzmann relation

$$
n=n_{0} \exp \left(\frac{e \phi-e \phi_{0}}{T_{e}}\right)
$$

Combining (2) and (5) gives

$$
\frac{d v}{d z}=\frac{e}{T_{e}} E v
$$

Combining with (1) gives

$$
v \frac{e}{T_{e}} E v=\frac{e E}{m}-\nu_{c} v
$$

so assuming that the ions reach the Bohm velocity $\mathrm{v}=\mathrm{c}_{\mathrm{s}}$ at the presheath/sheath boundary

$$
\begin{aligned}
v & =\frac{e}{m \nu} E\left(1-\frac{v^{2}}{c_{s}^{2}}\right) \\
& =\mu E\left(1-\frac{v^{2}}{c_{s}^{2}}\right)
\end{aligned}
$$

where $\mu \equiv e / m \nu$. If ionization is not neglected, (9) holds but $\mu_{c}$ is defined as in $e / m\left(\nu+\nu_{i}\right)$.

The effect of retaining the convective derivative is to add the factor $1-v^{2} / c_{s}^{2}$ in (8). This means that the ion velocity begins to deviate from the usual expression for mobility limited when $v / c_{s}$ exceeds 0.3 . Assuming no ionization, the presheath electric field is given by

$$
E=-\frac{T_{e}}{2 e} \sqrt{\frac{1}{\lambda_{c}\left(x-x_{0}\right)}} .
$$

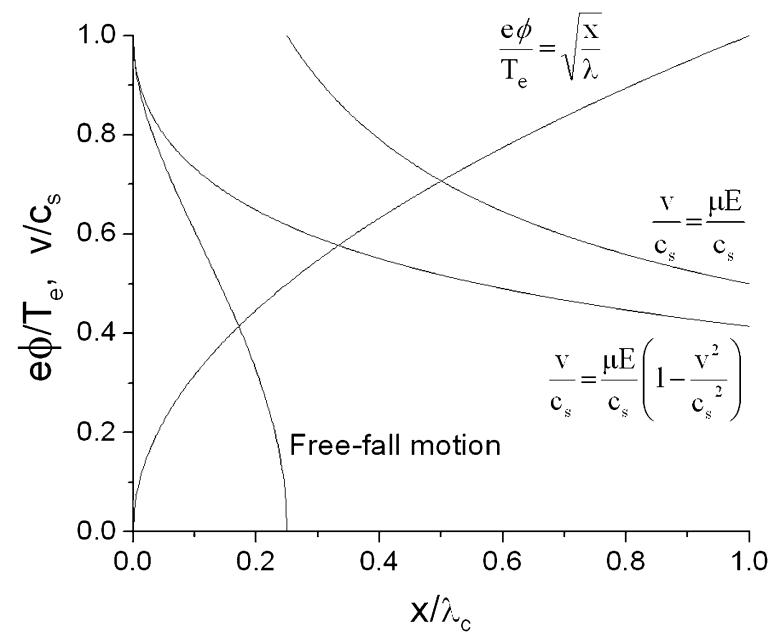

Fig. 1. Comparison of values of $e \phi / T_{e}$, and $v / c_{s}$ from (3), (4), (9), and (15).

Combining (8) and (10), and solving for $\mathrm{v}$

$$
v=-\frac{c_{s}^{2}}{2 \nu} \sqrt{\frac{1}{\lambda_{c}\left(x-x_{0}\right)}}\left(1-\frac{v^{2}}{c_{s}^{2}}\right)
$$

and

$$
\frac{v}{c_{s}}=-\frac{1}{2} \sqrt{\frac{\lambda_{c}}{\left(x-x_{0}\right)}}\left(1-\frac{v^{2}}{c_{s}^{2}}\right)
$$

where $\lambda_{c} \equiv c_{s} / \nu$.

Using (3), (12) becomes

$$
\frac{v}{c_{s}}=-\frac{1}{2} \frac{T_{e}}{e\left(\phi-\phi_{0}\right)}\left(1-\frac{v^{2}}{c_{s}^{2}}\right)
$$

As $v$ approaches $c_{s}$

$$
\begin{aligned}
\frac{v}{c_{s}} & \approx 1 \approx-\frac{m c_{s}^{2}}{2 e\left(\phi-\phi_{0}\right)}+\frac{m v^{2}}{2 e\left(\phi-\phi_{0}\right)} \\
e\left(\phi-\phi_{0}\right) & \approx-\frac{m c_{s}^{2}}{2}+\frac{m v^{2}}{2}
\end{aligned}
$$

Thus, free-fall ion motion is found close to the presheath/sheath boundary. Solving (9) for $\mathrm{v}$ gives:

$$
\begin{aligned}
2 \frac{v}{c_{s}} & =-\frac{c_{s}}{\mu E} \pm \sqrt{\frac{c_{s}^{2}}{\mu^{2} E^{2}}+4} \\
& =\frac{c_{s}}{\mu E}\left(-1 \pm \sqrt{1+4 \frac{\mu^{2} E^{2}}{c_{s}^{2}}}\right) .
\end{aligned}
$$

Fig. 1 gives a comparison of the (3), (4), (9), and (15). It is apparent that "free-fall" only describes the ion motion for velocities greater than $90 \%$ of the Bohm velocity, and this occurs very close to the presheath/sheath boundary, where the convective term in (7) is approximately five times greater than the collisional term. 


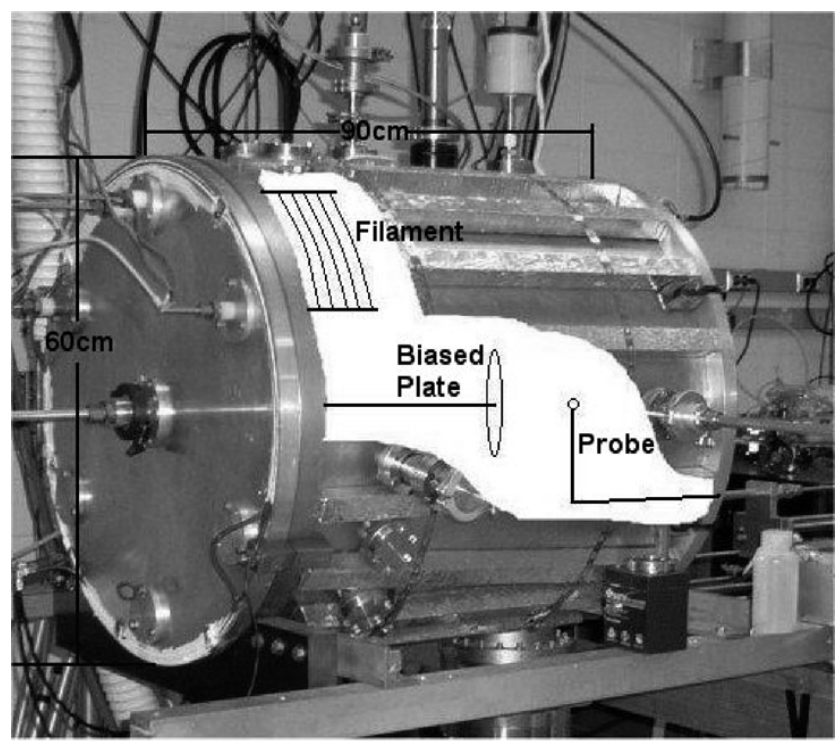

Fig. 2. Hot filament-produced multidipole plasma system.

\section{EXPERIMENTAL CONSIDERATIONS}

Presheath data were obtained in hot filament-produced multidipole plasma (see Fig. 2) operating with argon or mixtures of argon and helium [10]. Note that magnets were only located on the side walls. The electron temperature $T_{e}$ was found with Langmuir probes to be approximately $3 \mathrm{eV}$. The ion temperature $T_{i}$ determined from laser induced fluorescence (LIF) data was found to be close to room temperature in the bulk plasma so $\mathrm{T}_{\mathrm{i}} \ll<\mathrm{T}_{\mathrm{e}}$. Plasma potential was measured with emissive probes operating in the limit of zero emission [11]. This technique has a plasma potential resolution of $0.2 \mathrm{~V}$ and a spatial resolution of $1 \mathrm{~mm}$. Axial potential profiles were measured along the axis of a 14-cm diameter stainless steel plate mounted $43 \mathrm{~cm}$ from the end wall.

The relative size of the collision frequency and the ionization rate can be estimated as follows. Balancing plasma production with ion loss gives

$$
n \nu_{i} V \approx 0.6 \times 2 n c_{s} A
$$

where $A$ is the loss area, and $V$ is approximately equal to chamber length $L$ multiplied by end wall area $A_{w}$. Assuming the multidipole fields significantly reduce the loss to side walls, the loss area can be estimated to be approximately the area of the end walls $2 A_{w}$. Equation (17) then reduces to

$$
\nu_{i}=\frac{c_{s}}{L} .
$$

The ion-neutral collision frequency is the order of $\nu_{c}=c_{s} / \lambda$, so

$$
\frac{\nu_{i}}{\nu_{c}}=\frac{\lambda}{L}
$$

The collision length was chosen to be small compared to the chamber length by operating at a sufficiently high neutral pressure. Under these circumstances, we found that the profile of plasma potential [4] satisfied the (3) .

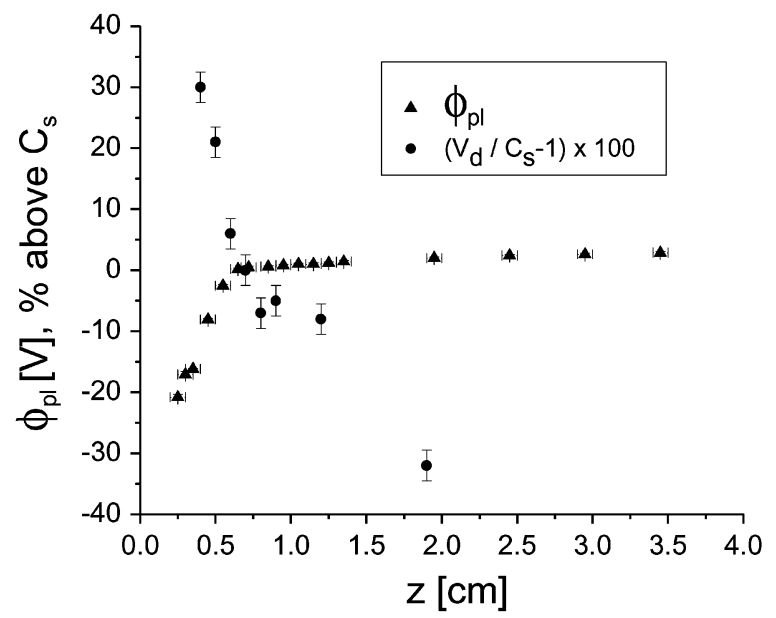

Fig. 3. Spatial profiles of the plasma potential and ion drift velocity in $\mathrm{Ar}$ plasma. $C_{s}$ is the argon ion Bohm velocity.

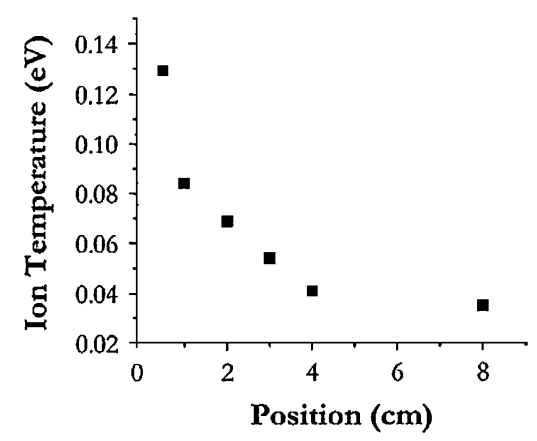

Fig. 4. Parallel ion temperature $\left(T_{i \|}\right)$ profile.

\section{A. Single Ion Species}

Laser induced fluorescence (LIF) measurements of the ion velocity distribution functions in single species (argon) plasmas demonstrated that the ions reach the Bohm velocity at the sheath edge [12], [13]. This expected result is shown in Fig. 3. A somewhat surprising result was that the ion temperature increased as the ions approached the plate [12], [14]. The ion temperature parallel to the normal to the plate $T_{i \|}$ was found to increase from approximately room temperature to $0.1 \mathrm{eV}$ in the presheath of a 0.6 mtorr argon plasma (see Fig. 4). The temperature increases because ions are born at room temperature from charge exchange and electron ionization over the presheath. These ions accelerate in the local electric field resulting in a significant spread in parallel ion velocity, i.e., a temperature increase, near the plate.

The ion temperature $T_{i \perp}$, perpendicular to the normal to the plate, was measured in ECR generated $N_{2}$ plasma [15]. In that case, a permanent magnetic field, expanding from the resonance region to the plate was also present. $T_{i \perp}$ was found to increase from room temperature in the expanding magnetic field to $0.12 \mathrm{eV}$ at 4 mtorr and $0.25 \mathrm{eV}$ at 0.5 mtorr. Here, a combination of ion scattering and charge exchange and ion acceleration by the ambipolar electric field is likely to be responsible for the temperature increase. 


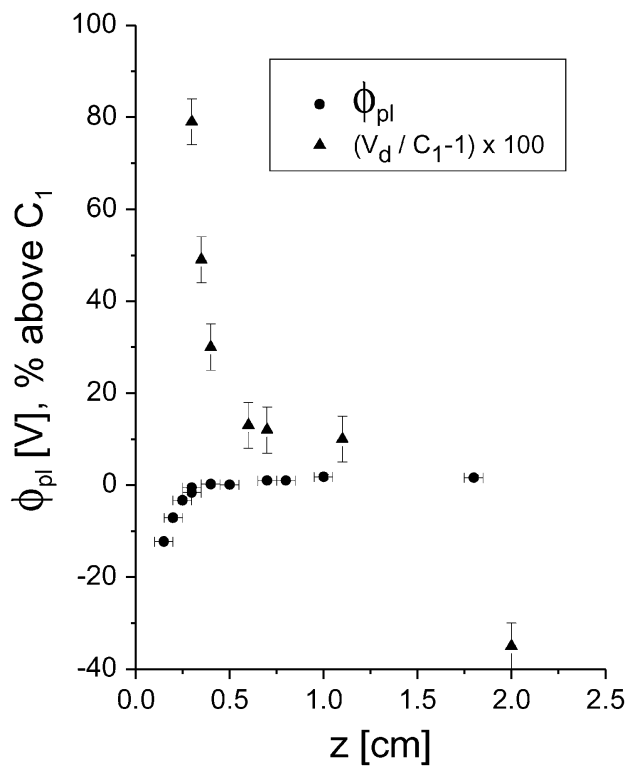

Fig. 5. Plasma potential and drift velocity profile for $\mathrm{Ar}^{+}$in an $\mathrm{Ar}+\mathrm{He}$ plasma. $\mathrm{C}_{1}$ is the argon ion Bohm velocity.

\section{B. Two Ion Species}

Most plasmas employed in industrial applications consist of more than one ion species. For example, $\mathrm{CF}_{2}^{+}, \mathrm{CF}_{3}^{+}, \mathrm{C}_{2} \mathrm{~F}_{4}^{+}$, and $\mathrm{C}_{2} \mathrm{~F}_{5}^{+}$are among the species found in $\mathrm{C}_{2} \mathrm{~F}_{6}$ etching of $\mathrm{SiO}_{2}$ [16].

1) Velocities at the Presheath/Sheath Boundary: The behavior of plasmas with two positive ion species is not a simple extrapolation from single positive ion species plasma. Mobility limited flow cannot provide the Bohm velocity or close to the Bohm velocity for each species. Nor is it clear that each ion species should reach those velocities. Riemann has derived a generalized Bohm condition [17]

$$
1 \geq \sum_{j} \frac{\left(n_{j 0} / n_{e 0}\right) c_{j}^{2}}{v_{j}^{2}}
$$

that must be satisfied at the presheath/sheath boundary by Boltzmann electrons. In (20), $n_{j 0} / n_{e 0}$ is the fractional density of $j$ th ion species, $c_{j}$ gives the individual species Bohm velocity, and $v_{j}$ is the individual species drift velocity at presheath/sheath boundary. The presheath/sheath boundary was identified as the position where a significant deviation from charge neutrality begins. For two species or more, choosing the equality in (20) does not uniquely determine the velocity of either species. Significant neutral density is present of both ion species, so charge exchange is present because of the low fractional ionization. Thus, it is difficult to determine the ion concentrations and velocities. LIF measurements of the argon ion velocity distribution function in a two species (argon + helium) plasma demonstrated that the $\mathrm{Ar}^{+}$ions reach their Bohm velocity before the sheath edge as shown in Fig. 5, [12]. The presheath/sheath boundary was identified from the plasma potential profile determined with emissive probes. The generalized Bohm condition could then be satisfied with $\mathrm{He}^{+}$ions moving slower than their Bohm velocity at the sheath boundary.

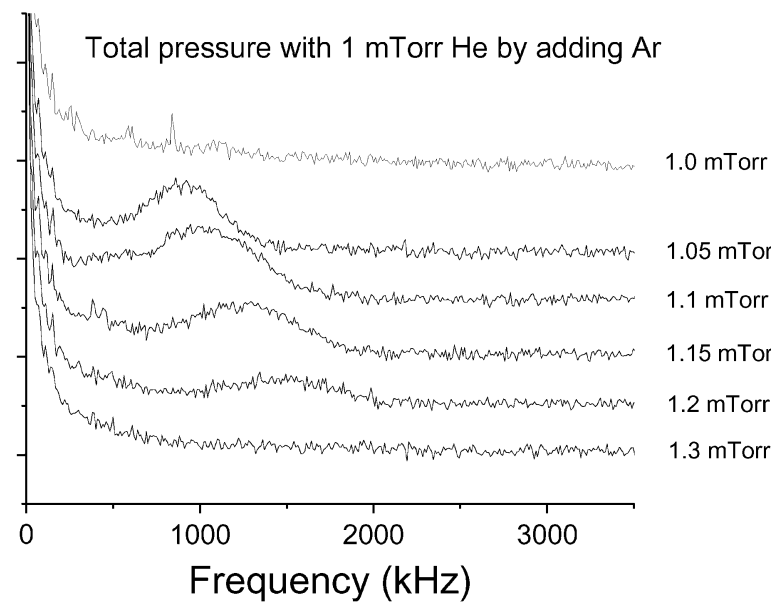

Fig. 6. Observed log of power spectra measured from ion saturation current as a function of the addition of Ar to a 1 mtorr He plasma. Curves are displaced vertically for clarity.

2) Instabilities: The ion velocity distribution function in the presheath consists of two ion beams with different velocities. The velocities differ by less than their individual Bohm velocities. Examination of the ion fluid equations shows that this distribution function is ion-ion two-stream unstable. Ion saturation current to a negatively biased cylindrical probe was analyzed with a spectrum analyzer. The frequency spectra are shown in Figs. 6 and 7 as a function of the neutral concentration of Ar added to a He plasma. As seen in Fig. 7(a), the instability frequency increases as the concentration of the heavier argon ion is increased. This behavior is qualitatively consistent with the predictions of the appropriate ion fluid and kinetic equations shown in Fig. 7(b). The linear growth rates of the instabilities shown in Fig. 7(b) are relatively small. It is important to note that the $\mathrm{Ar}^{+} / \mathrm{He}^{+}$ratio does not equal the $\mathrm{Ar} / \mathrm{He}$ ratio because of Penning ionization of the $\mathrm{Ar}$ via $\mathrm{He}$ metastables [18]. The $\mathrm{Ar}^{+}$and $\mathrm{He}^{+}$concentrations were determined from the phase velocity of waves launched in the bulk plasma region [10], and not corrected for the effects of ion acceleration or charge exchange.

\section{DISCUSSION}

Presheaths are a characteristic of weakly ionized plasma. They were identified as the region where ions are accelerated to the appropriate velocity at the sheath/presheath boundary. In this paper, it is shown that presheaths are a rich environment in which a variety of phenomena take place, and in many cases their properties are not yet understood.

Presheaths do much more than accelerate ions. For single ion species plasma, the velocity at the presheath/sheath boundary in weakly collisional plasma is the Bohm velocity. Charge exchange dominates the details of the IEDF at the presheath/sheath boundary. Data show that the plasma potential is given by the square root of $x / \lambda$ in one species plasma. Mobility-limited flow describes ion motion for velocities small compared to the Bohm velocity. $T_{i \|}$ is found to increase as ions approach the boundary because of the creation of cold ions throughout the presheath. $T_{i \perp}$ is also found to increase, probably as a result of collisions 




(a)

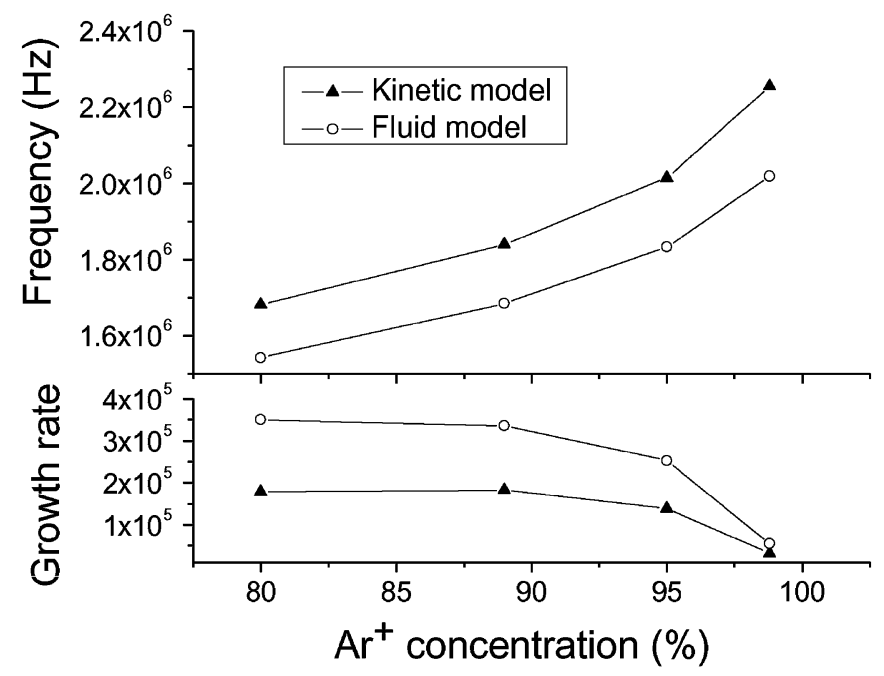

(b)

Fig. 7. (a) Peak frequency observed in Fig. 6 versus $\mathrm{Ar}^{+}$concentration. (b) Predicted frequencies and growth rates from fluid and kinetic equations.

of ions accelerated by the presheath electric field with background neutrals. Thus $T_{i \perp}$ is expected to depend on $T_{e}$ since the presheath potential drop depends on $T_{e}$. While the increase in $T_{i \perp}$ is small compared to $T_{e}$, it can be important. An especially important example is plasma etching of trenches and vias in semiconductors. Such etching requires ion anisotropy. The spread in perpendicular ion energy indicated by the $T_{i \perp}$ provides the ultimate limit to ion anisotropy.

For plasmas with two or more ion species, the ion velocities at the presheath/sheath boundary in weakly collisional plasma are somewhat different than the individual Bohm velocities. A generalized Bohm condition has been derived by Riemann [9] but it does not determine the individual species velocities nor has it been verified experimentally. Charge exchange dominates the details of the IEDF at the presheath/sheath boundary as it does for single species plasmas but the situation is much more complicated. Both symmetric and asymmetric charge exchange are present. Mobility limited flow, complicated by asymmetric charge exchange that mixes the ion species, describes ion motion for velocities small compared to the Bohm velocity. The $T_{i \|}$ of the $\mathrm{Ar}^{+}$in an $\mathrm{Ar}^{+}-\mathrm{He}^{+}$plasma is also found to increase as ions approach the boundary. In addition, the two species plasma is found to be ion-ion beam unstable. This provides a mechanism for energy exchange between the two ion species that could contribute to the speeding up the argon ions observed by LIF.

Overall, the presheath in weakly collisional unmagnetized plasmas is a busy environment. Single species presheath properties are generally understood. Multi-ion species presheaths are not well understood. Unfortunately, most presheaths of interest are at the boundaries of multi-ion species.

\section{REFERENCES}

[1] I. Langmuir, "The interaction of electron and positive ion space charge in cathode sheaths," Phys. Rev., vol. 33, pp. 954-989, June 1929.

[2] D. Bohm, The Characteristics of Electrical Discharges in Magnetic Field, A. Guthrie and R. K. Wakerling, Eds. New York: McGraw-Hill, 1949, ch. 3.

[3] M. A. Lieberman and A. J. Lichtenberg, Principles of Plasma Discharges and Materials Processing. New York: Wiley, 1994.

[4] L. Oksuz and N. Hershkowitz, "First experimental measurements of the plasma potential throughout the presheath and sheath at a boundary in a weakly collisional plasma," Phys. Rev. Lett., vol. 89, Sep. 2002.

[5] R. N. Franklin, "Plasmas with more than one species of positive ion and the Bohm criterion," J. Phys. D, vol. 33, pp. 3186-3189, Dec. 2000.

[6] _ - "The transition from collisionless to collisional in active low-temperature plasmas with more than one species of positive ion generated by electron impact and the Bohm criterion," J. Phys. D, vol. 34, pp. 1959-1962, Jul. 2001.

[7] K. F. Stephens II and C. A. Ordonez, "Sheath and presheath potentials for anode, cathode and floating plasma-facing surfaces," J. Appl. Phys., vol. 85, pp. 2522-2527, Mar. 1999.

[8] D. J. Koch and W. N. G. Hitchon, "The effects of collisions on the plasma presheath," Phys. Fluids. B, vol. 1, pp. 2239-2243, Nov. 1989.

[9] K. U. Riemann, "The influence of collisions on the plasma sheath transition," Phys. Plasmas, vol. 4, pp. 4158-4164, Nov. 1997.

[10] A. M. Hala and N. Herskowitz, "Ion acoustic wave velocity measurement of the concentration of two ion species in a multidipole plasma," Rev. Sci. Instrum., vol. 72, pp. 2279-2281, May 2001.

[11] J. R. Smith, N. Hershkowitz, and P. Coakley, "Inflection-point method of interpreting emissive probe characteristics," Rev. Sci. Instrum., vol. 50, pp. 210-218, Feb. 1979.

[12] L. Oksuz, M. A. Khedr, and N. Hershkowitz, "Laser induced fluorescence of argon ions in a plasma presheath," Phys. Plasmas, vol. 8, pp. 1729-1733, May 2001.

[13] G. D. Severn, X. Wang, E. Ko, and N. Hershkowitz, "Experimental studies of the Bohm criterion in a two-ion-species plasma using laser-induced fluorescence," Phys. Rev. Lett., vol. 90, no. 14, Apr. 2003. Art. no. 145001 .

[14] S. L. Gulick, B. L. Stansfield, Z. Abouassaleh, C. Boucher, J. P. Matte, T. W. Johnston, and R. Marchand, "Measurement of pre-sheath flow velocities by laser-induced fluorescence," J. Nucl. Mater, vol. 176, pp. 1059-1063, Dec. 1990.

[15] E. A. Den Hartog, H. Persing, and R. C. Woods, "Laser-induced fluorescence measurements of transverse ion temperature in an electron-cyclotron resonance plasma," Appl. Phys. Lett., vol. 57, no. 7, pp. 661-663, Aug. 1990.

[16] D. Zhang and M. J. Kushner, "Investigations of surface reactions during $\mathrm{C}_{2} \mathrm{~F}_{6}$ plasma etching of $\mathrm{SiO}_{2}$ with equipment and feature scale models," J. Vac. Sci. Tech. A, vol. 19, no. 2, pp. 524-538, Mar. 2001.

[17] K. U. Riemann, "The Bohm criterion and boundary conditions for a multicomponent system," IEEE Trans. Plasma Sci., vol. 23, no. 4, pp. 709-716, Aug. 1995.

[18] G. Hairpetian and R. L. Stenzel, "Mass-sensitive ion energy analyzer for multispecies plasmas," Rev. Sci. Instrum., vol. 58, pp. 2099-2102, Nov. 1987. 


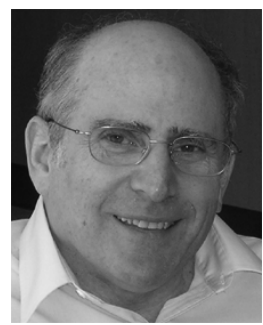

Noah Hershkowitz (SM'82-F'89) received the B.S. degree in physics from Union College, Schenectady, NY, in 1962 and the Ph.D. degree in physics from Johns Hopkins University, Baltimore, MD, in 1966.

$\mathrm{He}$ is the Irving Langmuir Professor of Engineering Physics at the University of Wisconsin, Madison. He was a Professor of Physics at the University of Iowa, Iowa City, from 1967 to 1981, and a Visiting Professor at the University of California at Los Angeles, from 1974 to 1975 and the University of Colorado, Boulder, from 1980 to 1981, before taking up his present position in 1981. Fourty-six Ph.D. students have completed their Ph.D. degrees under his direction. He has authored or coauthored more than 225 journal articles and is recognized as a world expert in experimental measurements of solitons, plasma sheaths, double layers, magnetic cusps, Langmuir probes, emissive probes, ICRF heating and ponderomotive stabilization of MHD instabilities in mirror-confined plasma, ICRF current drive in tokamaks, plasma etching and deposition, and basic plasma physics.

Prof. Hershkowitz was Chair of the American Physical Society Division of Plasma Physics (APS-DPP) (1990), Chair of the IEEE Plasma Science and Application Committee (PSAC) Executive Committee (1997-99), and President of the University Fusion Association (1985). He was Chairman of the Nuclear and Plasma Sciences Society (NPSS) Awards Committee and served on the NPSS Administrative Committee. He is a Fellow of the APS (1981), AVS (1999), and IOP (U.K.) (2001). He received the IEEE NPSS Merit Award (1987), the IEEE PSAC Award (1993), the Byron Bird Award for Excellence in a Research Publication (UW Madison) (1994), the AVS Plasma Prize (2004) and the APS-DPP Maxwell Prize (2004). He is founder and Editor-in-Chief of Plasma Sources Science and Technology (1991 to present). He was an Associate Editor of Physics Fluids (1981-1983) and Phyics Review Letters (1987-1990).

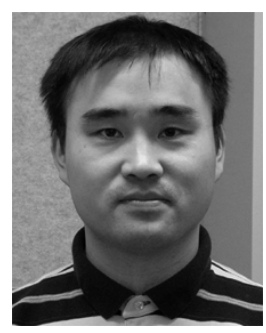

Eunsuk Ko (S'04) received the B.S. degree in physics from Yonsei University, Seoul, Korea in 1993, the M.S. degree in mechanical engineering, and the M.S. degree in engineering physics, in 1999 and 2001, respectively, from the University of Wisconsin, Madison, where he is currently working toward the $\mathrm{Ph} . \mathrm{D}$. degree. His graduate work involved in the development of uniform large area plasma, deep silicon, and metal etching. His Ph.D. research topic is the study of Mach probes in unmagnetized plasma.

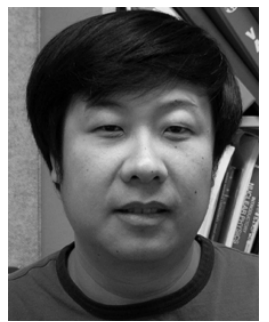

Xu Wang (S'04) received the B.S. degree in electrical engineering from the University of Electronic Science and Technology, Chengdu, China, in 1994 and the M.S. degree in engineering physics from the University of Wisconsin, Madison, in 2003, where he is currently working toward the Ph.D. degree in the Department of Engineering Physics. His dissertation work includes studies of the two-ion flow in plasma presheaths and instabilities excited by this two-ion flow near plasma boundaries.



Ahmed M. A. Hala was born in Madinah, Saudi Arabia, in 1972. He received the B.S. degree in nuclear engineering from King Abdulaziz University, Jeddah, Saudi Arabia, in 1994. He received the M.S. and Ph.D. degrees from the Nuclear Engineering and Engineering Physics Department, the University of Wisconsin, Madison, in 1995, and 2000, respectively. His Ph.D. dissertation included the discovery of a natural phenomenon in plasma physics (two-ion beam instability in plasma presheath).

In 2001 he was appointed as a Research Assistant Professor at the Space Research Institute (SRI) of King Abdulaziz City for Science and Technology (KACST), Riyadh, Saudi Arabia. At SRI, he is the Head of the advanced materials technology center. His research interests include basic plasma physics and plasma source ion implantation. 